\title{
Effect of Partially Hydrolyzed Ginsenoside on In Vitro Skin Permeation and Retention of Collagen Pentapeptide (Palmitoyl-KTTKS)
}

\author{
K. SELVARAJ, D. C. SHIN ${ }^{1}$ AND B. K. YOO* \\ College of Pharmacy, Gachon University, 191 Hambakmoero, Yeonsu-gu, Incheon 21936, ${ }^{1}$ Department of Skin Care R\&D, \\ Englewood Lab Korea, 425 Beongil-18 Namdongdaero, Namdong-gu, Incheon 21629, South Korea
}

Selvaraj et al.: Skin Permeation and Retention of Palmitoyl-KTTKS

\begin{abstract}
Although palmitoyl lysine threonine threonine lysine serine is claimed to be a useful bioactive peptide molecule to arrest skin aging, literature on transdermal permeation and retention of the peptide has rarely been reported. Aim of this study was to investigate the effect of partially hydrolyzed ginsenoside on transdermal permeation and retention of palmitoyl lysine threonine threonine lysine serine loaded liquid crystalline nanoparticles. Palmitoyl lysine threonine threonine lysine serine was loaded onto liquid crystalline nanoparticles using a hot melting and sonication method. Particle size of the liquid crystalline nanoparticles was reduced to $<\mathbf{3 0 0} \mathbf{~ n m}$ after addition of partially hydrolyzed ginsenoside into the constitution of the liquid crystalline nanoparticles and the polydispersity index indicated a monodisperse state. Transmission electron microscopy showed multilayered spherical liquid crystalline nanoparticles with dense core and corona like surface. Franz diffusion cell study demonstrated that skin permeation of palmitoyl lysine threonine threonine lysine serine was significantly increased when loaded onto liquid crystalline nanoparticles comprising partially hydrolyzed ginsenoside $(p<0.01)$, and that this was proportional to the amount of the ginsenoside added. The steady state flux of palmitoyl lysine threonine threonine lysine serine in liquid crystalline nanoparticles comprising $2.84 \%$ ginsenoside increased 4.60 fold and 2.36 fold compared to that of control (solubilized in propylene glycol) and liquid crystalline nanoparticles without ginsenoside, respectively. Partially hydrolyzed ginsenoside may be useful as a novel material to improve skin permeation of peptide molecules suffering from low transdermal absorption.
\end{abstract}

Key words: Palmitoyl lysine threonine threonine lysine serine, liquid crystalline nanoparticle, partially hydrolyzed ginsenoside, skin permeation, skin retention

In recent years, a pentapeptide comprising lysinethreonine-threonine-lysine-serine (KTTKS) has attracted attention as a substance that may arrest or delay skin aging ${ }^{[1-3]}$. This signaling peptide promotes extracellular matrix production in subconfluent fibroblast cell cultures and loses this activity as cells become fully confluent ${ }^{[4,5]}$. Because of its specific stimulatory effects on type I and III collagens and fibronectin, it is claimed to be beneficial for the prevention of wrinkles caused by skin aging ${ }^{[6]}$.

However, permeation of KTTKS through skin's stratum corneum layer is limited when applied topically as it is very hydrophilic in nature $(\log \mathrm{p}=-3.27)$. Therefore, it is difficult to obtain physiologically effective levels of the pentapeptide in the skin tissue. In an attempt to overcome this skin permeation issue, a long chain fatty

*Address for correspondence

E-mail: byoo@gachon.ac.kr acid (palmitic acid) was introduced to the N-terminal group of the lysine residue in KTTKS, resulting in a new molecule called palmitoyl KTTKS (pal-KTTKS). A similar attempt was made by conjugating ascorbic acid to the pentapeptide using succinoyl linker molecules, yielding another new molecule (ascorbyl KTTKS) ${ }^{[7]}$.

Although these strategies are considered promising, the paucity of literature regarding the transdermal permeation and retention of KTTKS raises questions about the clinical benefits of its intended use. Indeed,

This is an open access article distributed under the terms of the Creative
Commons Attribution-NonCommercial-ShareAlike 3.0 License, which
allows others to remix, tweak, and build upon the work non-commercially,
as long as the author is credited and the new creations are licensed under
the identical terms

Accepted 22 February 2021

Revised 18 January 2021

Received 09 September 2020

Indian J Pharm Sci 2021;83(1):76-83 
the clinical benefits of the new and modified KTTKSrelated molecules were marginal in several human trials $^{[8-11]}$. Furthermore, the molecular weights of palmitoyl and ascorbyl KTTKS are 802.05 and 811.74 $\mathrm{g} / \mathrm{mol}$, respectively, which are too large for the prodrug type molecules to permeate the stratum corneum layer without the aid of transdermal delivery system technology.

In an attempt to design an appropriate transdermal delivery system, we formulated a liquid crystalline nanoparticle (LCN) dispersion loaded with various active pharmaceutical ingredients and demonstrated improved physicochemical properties such as improved long term stability ${ }^{[12-14]}$. Furthermore, transdermal permeation of active pharmaceutical ingredients such as finasteride and tacrolimus through the transdermal delivery system was significantly improved ${ }^{[15,16]}$.

In this study, we prepared pal-KTTKS loaded LCN comprising monoolein (MO), phosphatidylcholine and partially hyrolyzed ginsenoside, and investigated physicochemical properties of the LCN and the effects of the ginsenoside on the skin permeation profile of the pentapeptide. An in vitro skin retention study was also performed to identify whether the LCN improves skin retention of the pentapeptide.

\section{MATERIALS AND METHODS}

Pal-KTTKS was supplied by Bio FD\&C (Incheon, Korea). MO was obtained from Danisco Japan (Tokyo, Japan), and poloxamer 407 (P407) was purchased from BASF (Ludwigshafen, Germany). Phosphatidylcholine was purchased from Lipoid (Germany), and partially hydrolyzed ginsenoside was gifted by Suam Longevity (Seongnam, Korea). The partially hydrolyzed ginsenoside comprised $39.99 \% \mathrm{Rg} 1$ (one of the active compounds found in ginseng), $14.05 \% \mathrm{Rd}, 13.79 \%$ F2, $11.55 \%$ protopanaxadiol; $7.95 \%$ Rg3, $5.75 \%$ compound $\mathrm{K}, 1.83 \%$ protopanaxatriol, and other minor components. All other chemicals were of reagent grade and used without further purification.
Preparation of pal-KTTKS-loaded LCN dispersion:

LCNs loaded with pal-KTTKS were prepared using a melting and sonication method described previously with slight modification ${ }^{[12]}$. Briefly, MO, phosphatidylcholine, pal-KTTKS and partially hydrolyzed ginsenoside, were dissolved into ethanol at $60^{\circ}$ in a clean dry glass vial. Distilled water containing P407 $(1 \% \mathrm{w} / \mathrm{v})$ was heated to the same temperature in another vial and added dropwise to the lipid mixture, resulting in the formation of coarse dispersion. This dispersion was then vortexed for $1 \mathrm{~min}$ and subjected to sonication for $5 \mathrm{~min}$ in a bath type sonicator (Ultrasonic 8893; Cole-Parmer, Vernon Hills, IL, USA) at $42 \mathrm{kHz}$. The LCN dispersion thus prepared was stored at $4^{\circ}$ until further uses. The composition and concentration of palKTTKS in the dispersion are shown in Table 1. Control sample was also prepared by directly dissolving palKTTKS into propylene glycol (4324.32 ppm).

\section{Dynamic light scattering and zeta potential of pal- KTTKS-loaded LCN dispersion:}

The particle size distribution and polydispersity index (PDI) were determined using dynamic light scattering at a fixed angle of $90^{\circ}$ using a Zetasizer Nano S90 (Malvern, United Kingdom). The dispersion was suitably diluted with distilled water $(0.5 \% \mathrm{w} / \mathrm{v}$ solid content) and analyzed at $25^{\circ}$ using a disposable sizing cuvette. Data were collected after an equilibration time of 2 min and averaged over 15 measurements. The zeta potential of the LCN dispersion was analyzed using an electrophoretic light scattering method (Photal ELSZ1000ZS, Malvern, United Kingdom) with a large bore capillary cell $(4 \mathrm{~mm})$ and a field strength of $20 \mathrm{~V} / \mathrm{cm}$. Measurements were performed in triplicate at $25^{\circ}$.

\section{Entrapment efficiency (EE) of pal-KTTKS-loaded LCN dispersion:}

EE was determined using an ultrafiltration method. Briefly, $0.5 \mathrm{ml}$ pal-KTTKS-loaded LCN dispersion

TABLE 1: COMPOSITION OF PALMITOYL KTTKS-LOADED LIQUID CRYSTALLINE NANOPARTICLES DISPERSION

\begin{tabular}{|c|c|c|c|c|c|c|c|c|}
\hline \multirow[b]{2}{*}{$\begin{array}{l}\mathrm{LCN} \\
\text { code }\end{array}$} & \multicolumn{5}{|c|}{ Oil phase } & \multicolumn{2}{|c|}{ Water phase } & \multirow[b]{2}{*}{$\begin{array}{l}\text { Concentration of } \\
\text { pal-KTTKS (ppm) }\end{array}$} \\
\hline & $\begin{array}{c}\text { Partially } \\
\text { hydrolyzed } \\
\text { ginsenoside (g) }\end{array}$ & $\begin{array}{c}\text { Monoolein } \\
\text { (g) }\end{array}$ & $\begin{array}{l}\text { Phosphatidylcholine } \\
\text { (g) }\end{array}$ & $\begin{array}{l}\text { Pal- } \\
\text { KTTKS } \\
\text { (g) }\end{array}$ & $\begin{array}{l}\text { Ethanol } \\
\text { (g) }\end{array}$ & $\begin{array}{c}\text { Poloxamer } \\
407 \text { (g) }\end{array}$ & $\begin{array}{l}\text { Distilled } \\
\text { water (g) }\end{array}$ & \\
\hline $\mathrm{F} 1$ & - & 3.00 & 0.96 & 0.08 & 4.36 & 0.10 & 10.00 & 4324.32 \\
\hline $\mathrm{F} 2$ & 0.18 & 3.00 & 0.96 & 0.08 & 4.36 & 0.10 & 10.00 & 4282.67 \\
\hline F3 & 0.36 & 3.00 & 0.96 & 0.08 & 4.36 & 0.10 & 10.00 & 4241.78 \\
\hline $\mathrm{F} 4$ & 0.54 & 3.00 & 0.96 & 0.08 & 4.36 & 0.10 & 10.00 & 4201.68 \\
\hline
\end{tabular}

LCN: liquid crystalline nanoparticle; pal-KTTKS: palmitoyl KTTKS. Control sample was prepared by directly dissolving pal-KTTKS (0.08 g) into $18.5 \mathrm{~g}$ of propylene glycol 
was centrifuged at $4000 \times \mathrm{g}$ for $30 \mathrm{~min}$ at $4^{\circ}$ using an Amicon Ultra-4 (molecular weight cut-off: 10000 $\mathrm{g} / \mathrm{mol}$, Merck-Millipore, Burlington, MA, USA), and then unentrapped pal-KTTKS was measured by High Performance Liquid Chromatography (HPLC) analysis of the filtrate. The total amount of the palKTTKS present in the initial dispersion was measured after destruction of the LCN structure by 10x dilution with methanol. EE was calculated using the following equation: $\mathrm{EE}(\%)=100 \times\left(\mathrm{D}_{\text {total }}-\mathrm{D}_{\text {unentrapped }}\right) / \mathrm{D}_{\text {total }}$, where $\mathrm{D}_{\text {total }}$ and $\mathrm{D}_{\text {unentrapped }}$ are the total and unentrapped amounts of pal-KTTKS, respectively.

\section{Polarized light microscopy of pal-KTTKS-loaded LCN dispersion:}

Liquid crystal formation was investigated using a polarized light microscope equipped with a digital camera (Olympus BX41-P, Tokyo, Japan). A drop of the pal-KTTKS loaded LCN dispersion was placed onto a cover glass and observed under plane polarized light condition. Images were captured by the microscope at 200x magnification and the same image was captured again with light passing through the crossed polarizer, whose vibration direction was $90^{\circ}$ relative to the plane polarizer.

\section{Transmission electron microscopy (TEM) of pal- KTTKS-loaded LCN dispersion:}

The diluted LCN dispersion was negatively stained with phosphotungstic acid (1\% neutral solution) to improve contrast by staining the nanoparticles. The stained dispersion was transferred onto a carbon coated copper grid, excess dispersion retained on the grid was carefully removed, and the mesh was air dried at room temperature. Images of the morphological structures of the nanoparticle were captured at an accelerating voltage of $100 \mathrm{kV}$ using a TEM (Hitachi 7600; Hitachi Ltd., Tokyo, Japan)

\section{Fourier transforms infrared spectroscopy (FTIR):}

FTIR absorption spectra were utilized to evaluate the physicochemical interaction between the pal-KTTKS and adjuvants used in the formulation using rotrode filter spectroscopy-100 (RFS) Bruker FT spectrometer with a Nd:YAG laser at room temperature. Spectra were recorded from 800 to $4000 \mathrm{~cm}^{-1}$. The entire spectrum was the average of two repeated measurements of 200 scans at $2 \mathrm{~cm}^{-1}$ resolution. The laser power was maintained at $100 \mathrm{~mW}$ for all measurements and all spectra were normalized to a background air spectrum at the end of each scan. Spectra were recorded using a liquid nitrogen cooled Germanium detector.

\section{In vitro skin permeation and retention of pal- KTTKS-loaded LCN dispersion:}

In vitro skin permeation and retention studies were performed with full thickness porcine skin using a Franz diffusion cell. Porcine skin was obtained from a local abattoir and carefully dissected to ensure complete removal of the subcutaneous fat before storing at $-20^{\circ}$. The skin was mounted on a Franz diffusion cell (diffusion area: $2.2 \mathrm{~cm}^{2}$ ) with the stratum corneum layer facing the donor compartment and the dermis layer facing the receptor compartment, which was filled with $10 \mathrm{ml}$ phosphate buffered saline (PBS, $\mathrm{pH}$ 6.0). The medium was constantly stirred at $400 \mathrm{rpm}$ and maintained at a temperature of $32^{\circ}$.

Five hundred microliters of pal-KTTKS dispersion and PBS $(50: 50 \mathrm{v} / \mathrm{v})$ with or without pancreatic lipase stock $(1 \mathrm{mg} / \mathrm{ml})$ was placed onto the skin and sealed with parafilm to prevent evaporation. The amount of palKTTKS in the donor compartment ranged between 1.05 and $1.08 \mathrm{mg}$, and the lipase concentration was fixed to $40 \mathrm{ppm}$. Aliquots $(0.5 \mathrm{ml})$ were collected from the receptor compartment at predetermined time interval $(1,3,6$, and $12 \mathrm{~h})$ and replaced with the same volume of fresh medium. The amount of the pal-KTTKS permeated was determined via HPLC. Control samples were also tested using the same procedure.

For the skin retention study, the effective diffusion area of the mounted skin was removed after $12 \mathrm{~h}$ and washed with distilled water to remove remaining pal-KTTKS dispersion. The skin was minced using a scalpel and probe sonicated with methanol for $2 \mathrm{~min}$ at $40 \%$ amplitude and $2 \mathrm{~s}$ on the "on" and "off" pulse mode. The solution was then centrifuged at $14000 \mathrm{rpm}$ for 5 min. The clear supernatant was filtered using a $0.45 \mu \mathrm{m}$ membrane filter, and the amount of pal-KTTKS was measured via HPLC.

\section{HPLC:}

Pal-KTTKS assay was performed using an HPLC system (Shimadzu, Kyoto, Japan) equipped with an LC 20AD pump and an SPD 20A (photodiode Array Detector) ultraviolet-visible light (UV-Vis) detector at $214 \mathrm{~nm}$, using L-ascorbic acid as internal standard. Inertsil Octadecyl silica (ODS-3) column $(4.6 \times 150$ $\mathrm{mm}, 5 \mu \mathrm{m}$, GL Sciences, Torrance, CA, USA) was used, and the temperature was set to $25^{\circ} \mathrm{C}$. The mobile phase consisted of acetonitrile and distilled water $(50: 50 \mathrm{v} / \mathrm{v})$ 
containing $0.1 \%$ trifluoroacetic acid. Flow rate was 0.4 $\mathrm{mL} / \mathrm{min}$ and injection volume was $20 \mu \mathrm{l}$. The retention times of the internal standard and pal-KTTKS were 3.4 and $6.1 \mathrm{~min}$, respectively.

\section{RESULTS AND DISCUSSION}

Pal-KTTKS was successfully loaded onto LCNs using a hot melting and sonication method (fig. 1). Particles in the LCN dispersion were of submicron size, and the average particle size was $<300 \mathrm{~nm}$ when the partially hydrolyzed ginsenoside was added (Table 2). Particle size was remarkably reduced after the addition of partially hydrolyzed ginsenoside compared to that of LCNs prepared without the ginsenoside $(p<0.01)$, and PDI decreased to $<0.2$, indicating an almost monodisperse state. Both parameters were proportional to the amount of the ginsenoside added, suggesting the stabilizing effect of the partially hydrolyzed ginsenoside on the LCN dispersion as a result of its amphiphilic nature.

The EE of the pentapeptide was $>99 \%$ for all formulations, indicating that the pentapeptide was thermodynamically stable and had high affinity with MO, a major component of the LCN. Particle size of the LCN dispersion was submicron size, and zeta potential was approximately $-30 \mathrm{mV}$ for all formulations (Table 2). This result suggests that the surface of the particles in the LCN dispersion was positively charged by the trimethylammonium cation of phosphatidylcholine, and an electrical double layer was formed by counter anions in the dispersion medium. The charge of the electrical double layer was not significantly affected by the ginsenoside content in this study $(0-2.84 \% \mathrm{w} / \mathrm{v})$.

Plane polarized microscopic image of the pal-KTTKS loaded LCN dispersion droplet showed yellow brownish droplet, and cross polarized image showed droplet with bright green stripes (fig. 1 and fig. 2). Appearance of an image under cross polarized microscopy indicates that crystallinity is present in the specimen. Uneven color density appears to be due to miscellaneous ingredients in the partially hydrolyzed ginsenoside. The yellow brown color in plane polarized image was created by the partially hydrolyzed ginsenoside and phospholipid.

To investigate the surface morphology of the particles in the LCN dispersion, TEM images were captured. Optical microscopic and TEM images as well as particle size distribution in the dispersion are shown in fig. 3. Most particles were clustered together and some particles were in a discrete state. In earlier studies using MO-based LCNs, optical microscopy and TEM images showed cube and fan like shapes ${ }^{[13,16]}$. In earlier studies, we suggested that cube and fan like shapes represented cubosomes and hexosomes, respectively, and different shapes were produced by the effect of the coexisting fatty acids. In this study, we introduced phosphatidylcholine, partially hydrolyzed ginsenoside, and ethanol into the composition of LCN. Unlike previous studies, the nanoparticles in this study were multilayered and spherical in form, with dense core and corona like surface tetherings structure. Considering that phosphatidylcholine and partially hydrolyzed ginsenoside are amphiphilic in nature ${ }^{[17]}$, corona like tethering structures appear to represent these additives whereas the dense and dark core part is formed by $\mathrm{MO}$, a constitutional material for LCN.

FTIR spectra of pal-KTTKS and pal-KTTKSloaded LCN are shown in fig. 4. Pal-KTTKS showed characteristic peaks of peptide molecules at 3274, 1672 and $1629 \mathrm{~cm}^{-1}$, representing $\mathrm{N}-\mathrm{H}$ stretching, $\mathrm{C}=\mathrm{O}$ stretching and $\mathrm{N}-\mathrm{H}$ bending, respectively. The
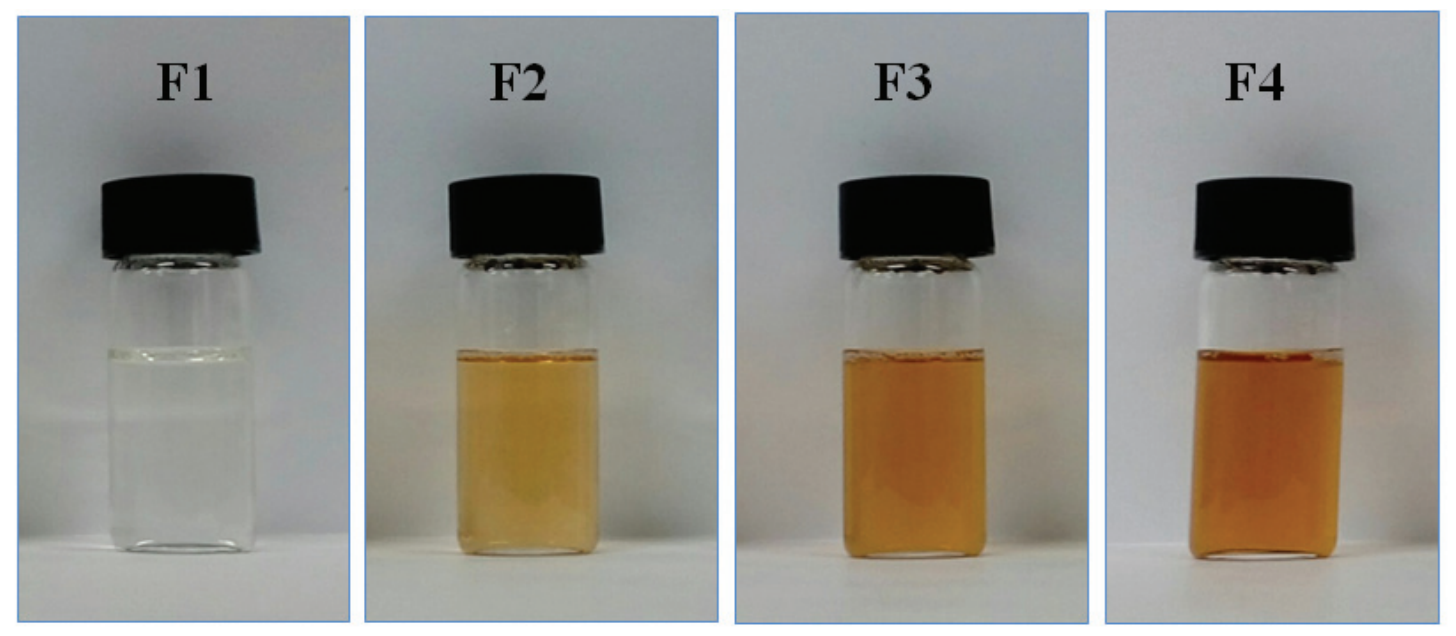

Fig. 1: Photographs of pal-KTTKS-loaded liquid crystalline nanoparticles dispersion prepared with different amount of partially hydrolyzed ginsenoside 
TABLE 2: CHARACTERIZATION OF PALMITOYL-KTTKS LOADED LIQUID CRYSTALLINE NANOPARTICLES DISPERSION

\begin{tabular}{lcccc}
\hline LCN code & EE $(\%)$ & Size $(\mathrm{nm})$ & PDI & Zeta potential $(\mathrm{mV})$ \\
\hline F1 & $99.87 \pm 0.02$ & $652 \pm 25.37$ & $0.426 \pm 0.162$ & $-30.42 \pm 0.42$ \\
F2 & $99.24 \pm 0.05$ & $286 \pm 16.14^{* *}$ & $0.216 \pm 0.027^{* *}$ & $-29.54 \pm 0.36$ \\
F3 & $99.85 \pm 0.03$ & $253 \pm 14.26^{* *}$ & $0.204 \pm 0.016^{* *}$ & $-32.30 \pm 0.62$ \\
F4 & $99.96 \pm 0.03$ & $207 \pm 18.12^{* *}$ & $0.202 \pm 0.023^{* *}$ & $-34.16 \pm 1.16$ \\
\hline
\end{tabular}

EE: entrapment efficiency; LCN: liquid crystalline nanoparticle; PDI: polydispersity index. " $\mathrm{p}<0.01$ compared to F1

TABLE 3: PERMEATION PARAMETERS OF PALMITOYL-KTTKS FROM THE PENTAPEPTIDE LOADED LIQUID CRYSTALLINE NANOPARTICLES ACROSS THE PORCINE SKIN

\begin{tabular}{|c|c|c|c|c|c|c|c|c|c|c|}
\hline \multirow[b]{2}{*}{$\begin{array}{l}\text { Release } \\
\text { parameter }\end{array}$} & \multicolumn{5}{|c|}{ Without lipase } & \multicolumn{5}{|c|}{ With lipase (40 ppm) } \\
\hline & Control & F1 & $\mathrm{F} 2$ & F3 & F4 & Control & $\mathrm{F} 1$ & $\mathrm{~F} 2$ & F3 & $\mathrm{F} 4$ \\
\hline $\mathrm{J}_{\mathrm{ss}}\left(\mu \mathrm{g} \times \mathrm{cm}^{-2} \times \mathrm{h}^{-1}\right)$ & $\begin{array}{c}2.537 \\
\pm 0.206^{\$ \$}\end{array}$ & $\begin{array}{c}4.935 \\
\pm 0.510^{* *}\end{array}$ & $\begin{array}{c}8.064 \\
\pm 0.316^{* *}, \$ \$\end{array}$ & $\begin{array}{c}7.991 \\
\pm 0.167^{* *}, \$ \$\end{array}$ & $\begin{array}{c}11.667 \\
\pm 1.525^{* *}, \$ \$\end{array}$ & $\begin{array}{c}2.537 \\
\pm 0.288^{\$ \$}\end{array}$ & $\begin{array}{c}6.317 \\
\pm 0.360^{* *}\end{array}$ & $\begin{array}{c}8.798 \\
\pm 0.239^{* *}, \$ \$\end{array}$ & $\begin{aligned} & 12.343 \\
\pm & 2.008^{* *}, \$ \$\end{aligned}$ & $\begin{array}{c}15.518 \\
\pm 1.984^{* *}, \$ \$\end{array}$ \\
\hline $\begin{array}{l}\text { Intercept } \\
\left(\mu \mathrm{g} \times \mathrm{cm}^{-2}\right)\end{array}$ & $\begin{array}{c}26.083 \\
\pm 2.582^{\$}\end{array}$ & $\begin{array}{c}32.959 \\
\pm 2.552^{*}\end{array}$ & $\begin{array}{r}32.559 \\
\pm 2.930^{*}\end{array}$ & $\begin{array}{c}58.04 \\
\pm 4.215^{* *}, \$ \$\end{array}$ & $\begin{aligned} & 58.165 \\
\pm & 4.669^{* *}, \$ \$\end{aligned}$ & $\begin{array}{l}26.083 \\
\pm 5.208\end{array}$ & $\begin{array}{l}34.254 \\
\pm 4.568\end{array}$ & $\begin{array}{l}34.061 \\
\pm 5.062\end{array}$ & $\begin{array}{l}31.134 \\
\pm 6.085\end{array}$ & $\begin{aligned} & 80.903 \\
\pm & 8.168^{* *}, \$ \$\end{aligned}$ \\
\hline
\end{tabular}

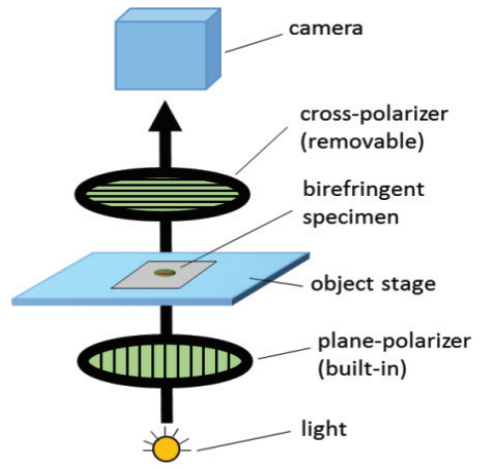

A

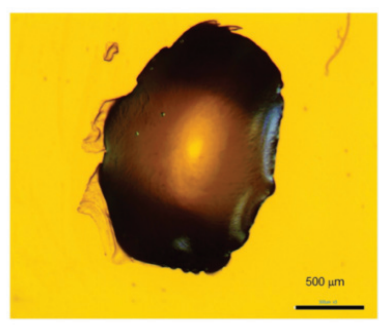

B

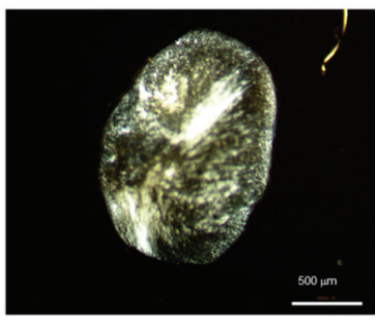

Fig. 2: Schematic representation of polarized microscope and images of pal-KTTKS loaded liquid crystalline nanoparticles dispersion

A: plane-polarizer image; B: cross-polarizer image
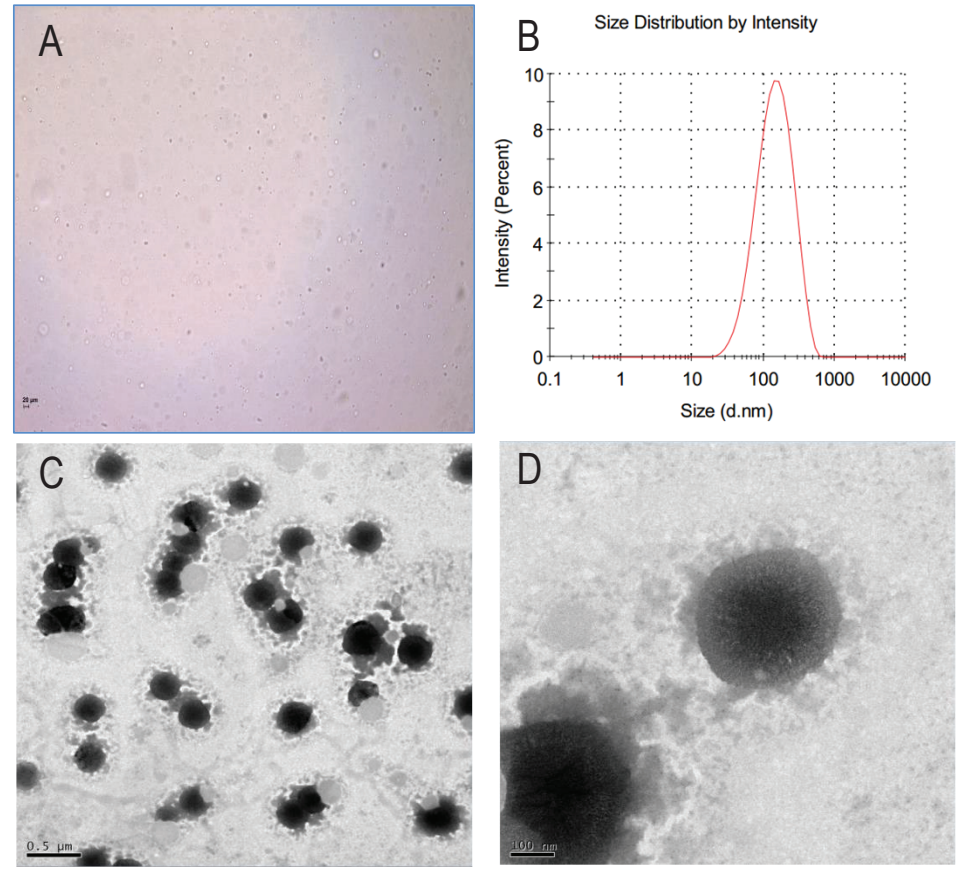

Fig. 3: Microscopic images of pal-KTTKS loaded liquid crystalline nanoparticles dispersion and its particle size distribution (F4 formula in Table 1)

A: optical microscope image (400x); B: particle size distribution; C: TEM image (low magnification); D: TEM image (high magnification) 
asymmetric and symmetric $\mathrm{C}-\mathrm{H}$ stretching of the methyl and methylene groups of $\mathrm{C} 16$ lipid chain (palmitoyl moiety) were observed at 2921 and $2852 \mathrm{~cm}^{-}$ 1, respectively. Pal-KTTKS-loaded LCN demonstrated broad band at $3392 \mathrm{~cm}^{-1}$, representing merged band of the hydroxyl group of $\mathrm{MO}$ and $\mathrm{N}-\mathrm{H}$ stretching of the pentapeptide. Peaks of $\mathrm{C}=\mathrm{O}$ stretching and $\mathrm{N}-\mathrm{H}$ bending of the pal-KTTKS were shifted toward slightly greater wave number whereas $\mathrm{C}-\mathrm{H}$ stretching of the aliphatic C16 lipid chain did not show any shift. FTIR spectra of the four formulations prepared in this study were identical although the amount of the partially hydrolyzed ginsenoside was different in the formulations.

A Franz diffusion cell study was carried out to evaluate how partially hydrolyzed ginsenoside affects the permeation and retention of the pentapeptide across porcine skin. The skin permeation and retention profiles of the peptide are depicted in fig. 5 and fig. 6, respectively, and permeation parameters are summarized in Table 3. The amount of the pentapeptide permeated through the skin and retained in the skin increased significantly when the peptide was loaded onto LCNs compared to that with propylene glycol solubilized pentapeptide (fig. 5 and fig. 6, $\mathrm{p}<0.01$ ). Interestingly, ginsenoside content in the LCNs was positively correlated with permeation and retention of pal-KTTKS. Similar results were obtained with regard to permeation and retention in the presence of lipase, suggesting that the amide bond in the peptide (between the palmitic acid moiety and the N-terminal of the lysine residue) is stable in the presence of lipase which is an enzyme in the skin. The steady state flux of the pentapeptide increased was 4.6 and 6.1 fold compared to that in the control (solubilized in propylene glycol) in the absence and presence of lipase, respectively

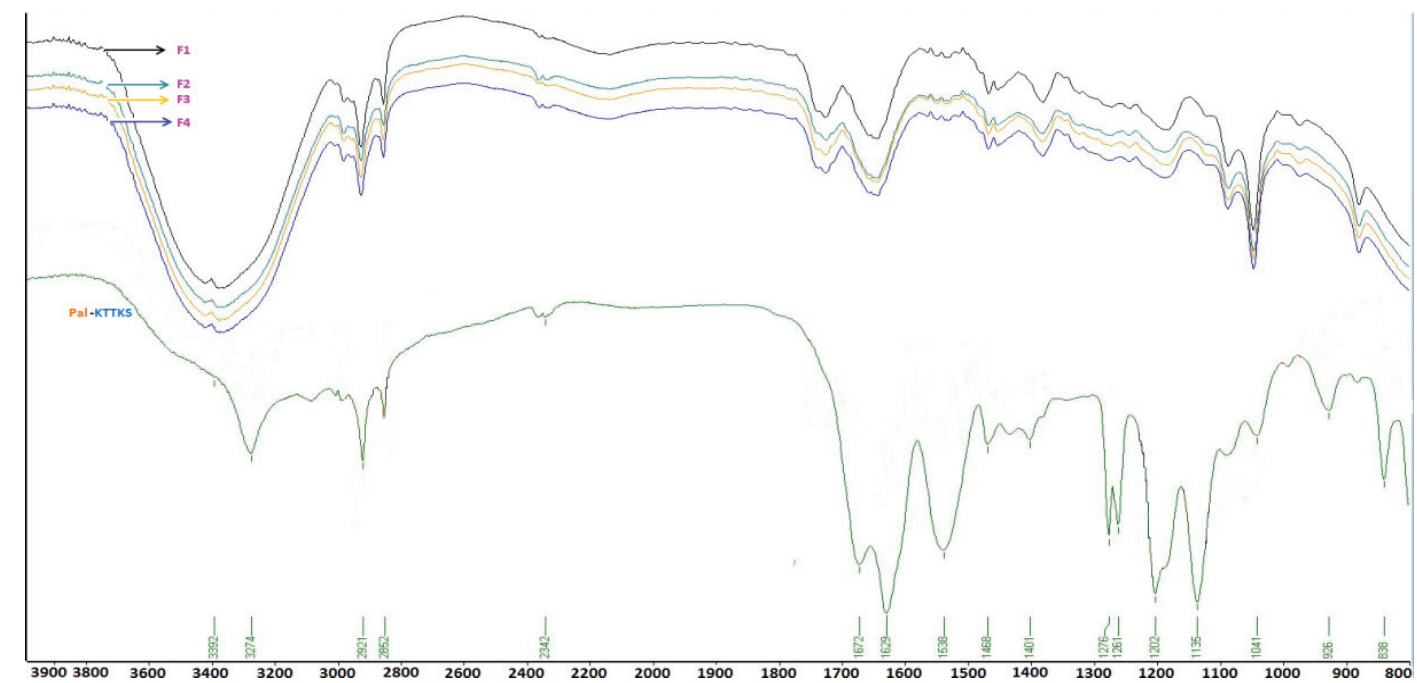

Fig. 4: FTIR spectrum of pal-KTTKS loaded liquid crystalline nanoparticles
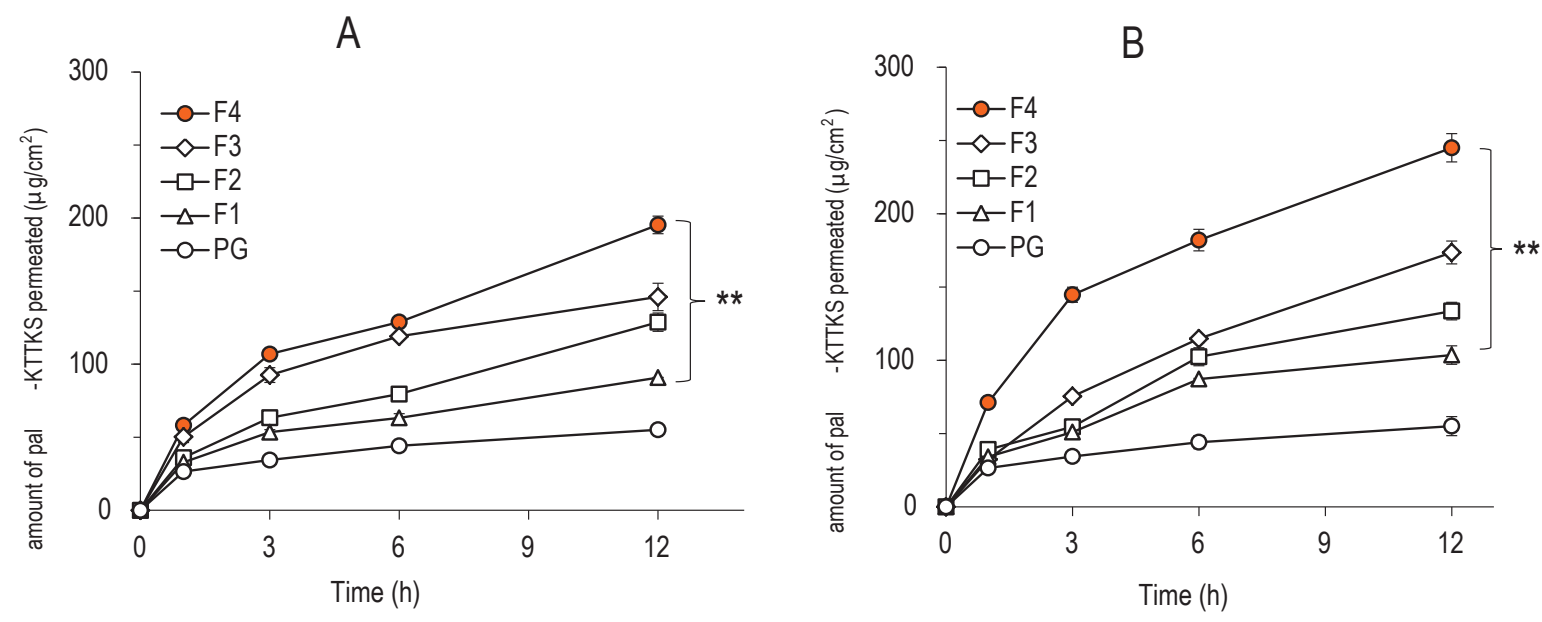

Fig. 5: Effect of partially hydrolyzed ginsenoside on the skin permeation of pal-KTTKS-loaded liquid crystalline nanoparticles dispersion A: without lipase; B: with lipase. **p<0.01 compared to PG 
A

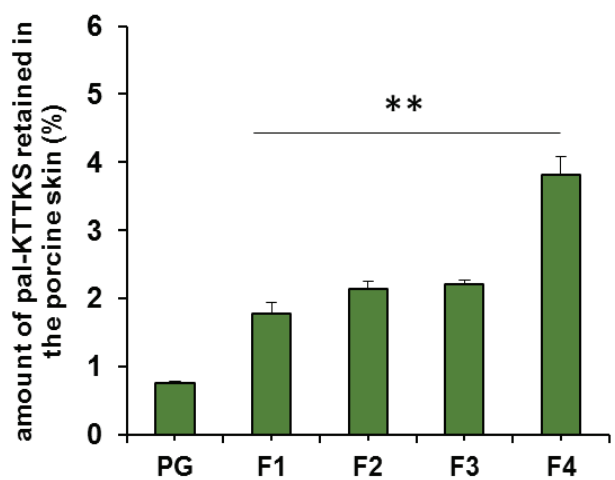

B

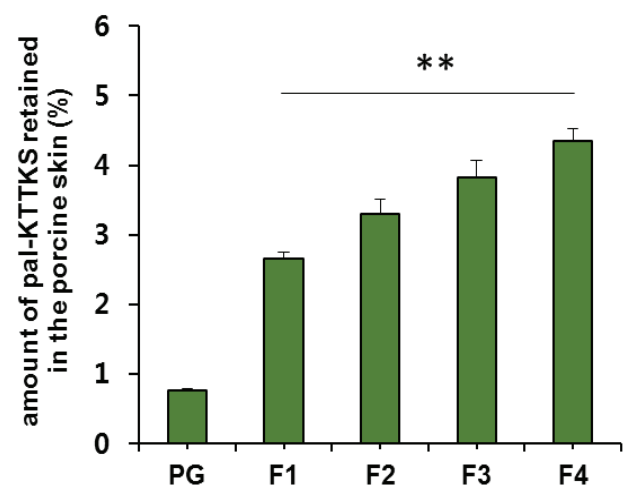

Fig. 6: Effect of partially hydrolyzed ginsenoside on the skin retention of pal-KTTKS-loaded liquid crystalline nanoparticles dispersion

A: without lipase; B: with lipase. ${ }^{* * p}<0.01$ compared to PG

(Table 3). These findings confirm that partially hydrolyzed ginsenoside significantly enhanced the permeation and retention profiles of the pentapeptide compared to those of simple solution or conventional LCN formulations.

Pal-KTTKS is a pentapeptide linked to palmitic acid to improve permeation of the KTTKS through the skin. It belongs to a signaling peptide which is capable of regulating cell proliferation and extracellular matrix regeneration by interacting with specific receptor. By regulating the extracellular matrix regeneration, palKTTKS has been claimed to be a useful bioactive peptide molecule for skin anti-aging ${ }^{[8,18,19]}$.

However, proteolytic and/or amidolytic activities of the skin may break down the pal-KTTKS during permeation process because skin also contains enzyme systems like other tissues. In this study, we anticipated that liquid crystallinity would help avoid the enzymatic activities of the skin. Normally, transparent liquids have one refractive index, and therefore, do not produce any images under cross polarized light. However, the palKTTKS loaded LCNs dispersion prepared in this study showed a distinct image under cross polarized light. This result indicates that, although the pal-KTTKS loaded LCN dispersion looks transparent in a liquid state, an anisotropic (birefringent) substance is in fact present as crystalline state.

Our results showed that significant amount of palKTTKS was detected in the receptor compartment of the Franz diffusion cell, indicating that proteolytic and amidolytic activities were successfully inhibited by applying the pentapeptide as LCN dispersion. The amount of pal-KTTKS detected in the receptor compartment at $12 \mathrm{~h}$ time point was $18.49,26.46$, 30.30 , and $40.91 \%$ of the total peptide for F1, F2, F3, and $\mathrm{F} 4$, respectively. It was only $11.22 \%$ when palKTTKS was directly solubilized into propylene glycol (PG) and applied on the skin. These results suggest that pal-KTTKS was either mostly broken down to amino acids or unable to permeate through the skin when applied as propylene glycol solubilized or LCN with less amount of the ginsenoside. Notably, addition of partially hydrolyzed ginsenoside was positively correlated with the skin permeation parameters in terms of flux and amount (Table 3, fig. 5 and fig. 6).

FTIR showed that peaks of $\mathrm{C}=\mathrm{O}$ stretching $\left(1672 \mathrm{~cm}^{-1}\right)$ and $\mathrm{N}-\mathrm{H}$ bending $\left(1629 \mathrm{~cm}^{-1}\right)$ of the pal-KTTKS were shifted toward slightly greater wave number. C-O stretching at $1136 \mathrm{~cm}^{-1}$ also shifted towards greater wave number. These findings suggest that peptide bonds in the pentapeptide appear to be hydrogen bonded with hydroxyl group of $\mathrm{MO}$, a constitutional material in the LCN. In contrast, C-H stretchings (2852 and $2921 \mathrm{~cm}^{-1}$ ) of the aliphatic C16 lipid chain stayed at the same wave number in all formulations, indicating that pal-KTTKS might have been entrapped in the water channels inside the MObased LCN via hydrogen bonding between the peptide moiety and hydroxyl group of the MO. Although the amount of the partially hydrolyzed ginsenoside was different, FTIR spectra of the four formulations were identical. This finding suggests that the ginsenoside did not interrupt entrapment of pentapeptide into water channels of the MO-based LCN.

Ginsenosides are a group of naturally occurring steroid glycosides which demonstrate a variety of physiological activities ${ }^{[20,21]}$. In this study, based on amphiphilic nature of the ginsenosides, we have speculated that they can also be used as solubilizer and permeability enhancer. However, there are few reports on the use of 
ginsenoside as solubilizer or permeability enhancer ${ }^{[22]}$. Recently, there are two articles published with regard to ginsenoside-based nanoparticle ${ }^{[17,23]}$. Ginsenoside used in the two reports was Rb1 which comprises four sugar molecules in a steroid skeleton. However, critical micelle concentration of the ginsenoside $\mathrm{Rb} 1$ is very low $(0.035 \%, \mathrm{w} / \mathrm{v})$, and therefore, $\mathrm{Rb} 1$ has limitations for use in industrial manufacturing processes because of too many bubbles. In contrast, the partially hydrolyzed ginsenoside used in this study is essentially hydrophobic, and therefore, it is very slightly soluble in water and does not form bubbles during manufacturing processes in aqueous environment. Hydrophobic property of the partially hydrolyzed ginsenoside provides additional benefit that it does not cause hemolysis which is detrimental issue for the most ginsenosides. Pal-KTTKS was successfully loaded onto MO-based LCNs with partially hydrolyzed ginsenoside. Our results demonstrated that ginsenoside significantly enhanced the skin permeation and retention profiles of the pentapeptide. This work highlights the potential of partially hydrolyzed ginsenoside to be used as a novel material to improve skin permeation of peptide molecules suffering from low transdermal absorption.

\section{Acknowledgements:}

This work was supported by an Industrial Cluster Competence Promotion Grant (1415158996) funded by the Korean Ministry of Trade, Industry and Energy (MOTIE).

\section{Conflict of interests:}

The authors declared no conflict of interest.

\section{REFERENCES}

1. Krishnamoorthy N, Tseng YT, Gajendrarao P, Sarathchandra P, McCormack A, Carubelli I, et al. A strategy to enhance secretion of extracellular matrix components by stem cells: relevance to tissue engineering. Tissue Eng Part A 2018;24(12):145-56.

2. Park H, An E, Lee AR. Effect of palmitoyl-pentapeptide (Pal-KTTKS) on wound contractile process in relation with connective tissue growth factor and $\alpha$-smooth muscle actin expression. Tissue Eng Regen Med 2017;14(1):73-80.

3. Guglielmi D, Martinelli A, Rissi N, Cilli EM, Soares CP, Chiavacci LA. Synthesis of the peptide Ac-Wahx-KTTKS and evaluation of the ability to induce in vitro collagen synthesis. Protein Pept Lett 2016;23(6):544-7.

4. Katayama K, Armendariz-Borunda J, Raghow R, Kang AH, Seyer JM. A pentapeptide from type I procollagen promotes extracellular matrix production. J Biol Chem 1993;268(14):9941-4.

5. Abu Samah NH, Heard CM. Topically applied KTTKS: a review. Int J Cosmet Sci 2011;33(6):483-90.
6. Namjoshi S, Caccetta R, Benson HA. Skin peptides: biological activity and therapeutic opportunities. J Pharm Sci 2008;97(7):2524-42.

7. Choi HI, Park JI, Kim HJ, Kim DW, Kim SS. A novel L-ascorbic acid and peptide conjugate with increased stability and collagen biosynthesis. BMB rep 2009;42(11):743-6.

8. Robinson LR, Fitzgerald NC, Doughty DG, Dawes NC, Berge CA, Bissett DL. Topical palmitoyl pentapeptide provides improvement in photoaged human facial skin 1. Int J Cosmet Sci 2005;27(3):155-60.

9. Lupo MP. Cosmeceutical peptides. Dermatol Surg 2005;31:832-6.

10. Pouillot A, Dayan N, Polla AS, Polla LL, Polla BS. The stratum corneum: a double paradox. J Cosmet Dermatol 2008;7(2):143-8.

11. Kaczvinsky JR, Griffiths CE, Schnicker MS, Li J. Efficacy of anti-aging products for periorbital wrinkles as measured by $3 \mathrm{D}$ imaging. J Cosmet Dermatol 2009;8(3):228-33.

12. Madheswaran T, Baskaran R, Sundaramoorthy P, Yoo BK. Enhanced skin permeation of $5 \alpha$-reductase inhibitors entrapped into surface-modified liquid crystalline nanoparticles. Arch Pharm Res 2015;38(4):534-42.

13. Thapa RK, Baskaran R, Madheswaran T, Kim JO, Yong CS, Yoo BK. Preparation, characterization, and release study of tacrolimus-loaded liquid crystalline nanoparticles. J Dispers Sci Technol 2013;34(1):72-7.

14. Thapa RK, Baskaran R, Madheswaran T, Rhyu JY, Kim JO, Yong CS, et al. Effect of saturated fatty acids on tacrolimusloaded liquid crystalline nanoparticles. J Drug Deliv Sci Technol 2013;23(2):137-41.

15. Madheswaran T, Baskaran R, Yoo BK, Kesharwani P. In vitro and in vivo skin distribution of $5 \alpha$-reductase inhibitors loaded into liquid crystalline nanoparticles. J Pharm Sci 2017; 106(11):3385-94.

16. Thapa RK, Baskaran R, Madheswaran T, Kim JO, Yong CS, Yoo BK. In vitro release and skin permeation of tacrolimus from monoolein-based liquid crystalline nanoparticles. J Drug Deliv Sci Technol 2012;22(6):479-84.

17. Dai L, Liu K, Si C, Wang L, Liu J, He J, et al. Ginsenoside nanoparticle: a new green drug delivery system. J Mater Chem B 2016;4(3):529-38.

18. Lintner K, Peschard O. Biologically active peptides: from a laboratory bench curiosity to a functional skin care product. Int J Cosmet Sci 2000;22(3):207-18.

19. Jones RR, Castelletto V, Connon CJ, Hamley IW. Collagen stimulating effect of peptide amphiphile C16-KTTKS on human fibroblasts. Mol Pharm 2013;10(3):1063-9.

20. Byeon SE, Choi WS, Hong EK, Lee J, Rhee MH, Park HJ, et al. Inhibitory effect of saponin fraction from Codonopsis lanceolata on immune cell-mediated inflammatory responses. Arch Pharm Res 2009;32(6):813-22.

21. Kim JS, Joo EJ, Chun J, Ha YW, Lee JH, Han Y, et al. Induction of apoptosis by ginsenoside Rk1 in SK-MEL-2human melanoma. Arch Pharm Res 2012;35(4):717-22.

22. Vijayakumar A, Baskaran R, Maeng HJ, Yoo BK. Ginsenoside improves physicochemical properties and bioavailability of curcumin-loaded nanostructured lipid carrier. Arch Pharm Res 2017;40(7):864-74.

23. Li M, Lan J, Li X, Xin M, Wang H, Zhang F, et al. Novel ultra-small micelles based on ginsenoside Rb1: a potential nanoplatform for ocular drug delivery. Drug Deliv 2019;26(1):481-9. 